\title{
The Role of Traffic Forecasting in QoS Routing - A Case Study of Time-Dependent Routing
}

\author{
Yuekang Yang Chung-Horng Lung \\ Department of Systems \& Engineering, Carleton University, Ottawa, Ontario, Canada \\ E-mail: \{yyang, chlung\}@sce.carleton.ca
}

\begin{abstract}
QoS routing solutions can be classified into two categories, state-dependent and time-dependent, according to their awareness of the future traffic demand in the network. Compared with representative state-dependent routing algorithms, a time-dependent variation of WSP - TDWSP - is proposed in this paper to study the role of traffic forecasting in QoS routing, by customizing itself for a range of traffic demands. Our simulation results confirm the feasibility of traffic forecasting in the context of QoS routing, which empowers TDWSP to achieve better routing performance and to overcome QoS routing difficulties, even though completely accurate traffic prediction is not required. The case study involving TDWSP further reveals that even a static forecast can remain effective over a large area in the solvable traffic demand space, if the network topology and the peak traffic value are given. Thus, the role of traffic forecasting in QoS routing becomes more prominent.
\end{abstract}

Keywords-QoS routing, time-dependent routing, forecast

\section{INTRODUCTION}

Over the past few decades, the topic of IP QoS routing has been discussed extensively, and related research has made a lot of progress. Nevertheless, in general, QoS routing still faces some primary challenges [1]:

1) Stability and Scalability. When multiple resources are allocated and de-allocated, a high frequency of state updates is required to avoid instability and route flapping [2, 3], but it does not scale well due to its high communication overhead for large networks. Unlike the cost in OSPF, the database of which is quasi-static, the cost of maintaining a synchronized QoS-database in a dynamic environment is high.

2) Robustness. Routers always get state updates with delays, and there is no guarantee that resource information is accurate and up-to-date. Route computation and routing decisions should be robust enough to be based on imprecise states [4].

3) Routing Cost. Processing state updates, implementing techniques related to the robustness issue, and conducting QoS routing all introduce considerable computational costs $[5,6]$. In contrast, QoS requests expect highly responsive service from the QoS routing algorithms.

\section{A. Motivation}

The primary motivation for this paper comes from Fig. 1, adopted from reference [7], which shows three typical traces of link traffic in the Sprint Internet core in May 2002.

Fig. 1 clearly shows that aggregated Internet traffic demonstrates strong predictability over a long period of time (i.e. hours, days and weeks). The two spikes between dotted lines represent Saturday and Sunday traffic, respectively, while the remaining periodic movement represents the weekday load. The daily traffic pattern between peak hours and normal hours is easily observed.
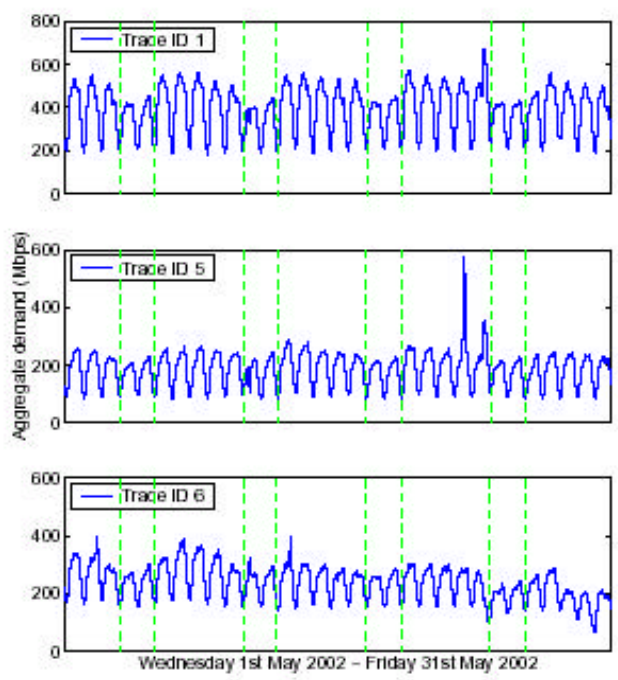

Fig. 1. Three traces of link traffic.

It is reasonable to believe that utilizing the knowledge of periodic traffic change may improve routing performance and relief challenges mentioned above. However, there is a concern that traffic forecasting is not reliable and could introduce significant additional cost to routing algorithms.

The goal of this paper is to answer the question whether the routing algorithm requires an accurate forecast. If the routing performance is sensitive to the forecast, then the traffic forecasting should be done carefully and frequently. Thus, the cost of forecasting could be high, and the benefit of traffic forecasting is fundamentally limited. On the other hand, if forecasting yields significantly better performance yet the routing algorithm does not need an accurate forecast, then we have the confirmation of the statement that traffic forecasting in QoS routing is beneficial and feasible.

\section{B. Scope}

Realizing that the QoS routing problem is intractable in many aspects, we limit the scope of this paper in order to provide us with some insights into the problem. The scope is three-fold: First, the paper assumes that aggregated Internet traffic has a periodic predictable pattern. The problem of traffic forecasting itself is not part of this paper. Second, link or node failure and traffic backup are not considered in this paper. Finally, it is assumed that the networks discussed in this paper fall under one single administration authority, or in one routing domain. 


\section{Our Contributions}

The contributions of this paper include:

1) Confirming that the combination of a priori knowledge of traffic demand and state-dependent routing algorithms would yield positive results, by proposing a time-dependent IP QoS Routing algorithm based on the Widest-Shortest Path (WSP) algorithm.

2) Identifying the feasibility of QoS routing based on a fixed traffic forecast. This paper does not deal with an adaptive forecasting schema that runs dynamically at reat time based on traffic conditions. Instead, the paper identifies that even a static piece of estimated information is valuable in QoS routing and can remain effective within a relatively wide range of traffic conditions.

3) Advocating the extreme traffic value forecast. From the results of the simulation, this paper discloses the fact that in terms of IP routing performance, the sensitivity of the forecast is asymmetrical. In the context of IP routing, this fact has numerous implications, which could be utilized not only for simplifying QoS routing algorithms, but also for collecting, analyzing and predicting traffic statistics.

The paper is structured as follows. Section II provides an overview of related research. Section III introduces methodologies of this work. Section IV proposes a timedependent routing algorithm. Section V presents simulation configurations. Section VI contains simulation results for the real traffic pattern, followed by section VII, which illustrates the results for artificial traffic patterns. The paper is concluded in section VIII with a summary of the results and highlights of the conclusions.

\section{RELATED WORK}

There is a large body of literature on QoS routing. One classification of QoS routing divides QoS routing algorithms into two categories: state-dependent and time-dependent. A routing algorithm that is able to find the optimal route for every QoS request, without knowing either the history or the future traffic demand, is called a state-dependent mechanism. Conversely, a routing algorithm that has knowledge of the history or future traffic demand is called a time-dependent mechanism [8].

Interestingly, most proposed QoS routing algorithms [9, $10,11]$ are state-dependent mechanisms. A minimal amount of research [12] has been done on the time-dependent category.

Widest-Shortest Path (WSP) and Shortest-Widest Path (SWP) [13] are variations of the Shortest Path (SP) algorithm adopted in PNNI. Realizing that SP selects a path arbitrarily from multiple paths with the same shortest distance (sum of link costs) and that this procedure is not fair enough, Guérin et al. modified the SP slightly to improve QoS routing performance. In the case of WSP, it picks the link with the largest residual link capacity among multiple shortest paths, if available, while for SWP, it picks the link with the shortest distance among multiple widest paths, if available. WSP is usually preferred, because the smallest link residual capacity is maximized.

The Minimum Interference Routing Algorithm (MIRA) proposed by Kodialam and Lakshman [14] addresses issues in more sophisticated scenarios when the selection of a path between a given source-destination pair can potentially influence many other pairs. They designed an algorithm to calculate the "interference" in order to avoid bottlenecks, and to select the best route for QoS requests.

Another, more recent work related to MIRA is ProfileBased Routing (PBR) [12], which applies traffic forecasting (i.e. a traffic profile) to improve QoS routing. When traffic profile changes at run time, PBR repeatedly generates preallocation plans based on a residual network that excludes occupied link bandwidth. The authors of [12] used a linear optimization method, rather than a nonlinear optimization one, which will be further discussed further in section III.

\section{METHODOLOGIES}

\section{A. Simplifications to QoS Constraints}

Typical QoS constraints include delay, packet loss, and bandwidth. The upper bounds of delay and loss for each link are adopted in this paper to simplify the delay and packet-loss constraints. The simplification is justified by the fact that the measured loss probabilities and delay for the same priority flow on different routers are of a similar order [17]. Meanwhile, bandwidth requirement can be simplified by using equivalent bandwidth technique $[18,19]$.

\section{B. Routing Performance Evaluation}

An optimal routing solution can be well represented by two objectives - minimizing average hop count and minimizing maximum link utilization. As proposed by Bonaventure et al. [17], a nonlinear objective function forms a compromise between the two previous objectives. However, it remains up to the ISP to decide which objective is more important, according to network operation status and specific requirements. More specifically, the objective function is shown in (1):

$$
\text { minimize } \sum_{e \in E}\left(1 / c_{e} \cdot \sum_{k \in K} x_{e, k}\right)^{n}, n \in[1, \infty)
$$

$E$ is the set of all edges, and $K$ is the set of all traffic flows. $c_{e}$ is the link capacity on edge $e$, while $x_{e, k}$ is the bandwidth of the $k$ th flow on edge $e$. The order $n$ is a useful parameter for ISPs to adjust the balance between two objectives.

In this paper, formula (1) is used in two ways: First, we use this formula as an objective function to calculate the optimal routing solution. Second, the value of (1) is regarded as being the level of network-wide performance when different QoS routing algorithms are compared.

\section{Traffic Demand Representation}

Let the traffic from node $i$ to destination node $j$ be $t_{i j}$. The traffic demand can then be represented in a vector form. An example of such a vector for a four-node network is:

$$
d=\left[t_{12}, t_{13}, t_{14}, t_{21}, t_{23}, t_{24}, t_{31}, t_{32}, t_{34}, t_{41}, t_{42}, t_{43}\right]
$$




\section{TIME-DEPENDENT WSP (TDWSP)}

TDWSP is a variation of WSP, but it knows the future traffic demand $d$ denoted as $f$. When $f$ is given, an optimum routing solution is calculated. Part of an example of such optimum solution ingressed from node 1 is depicted in Fig 2. For instance, there is a route, 1-5-6, going to node 6 , with a bandwidth of $24 \mathrm{Mbps}$. Subtract $3 \mathrm{Mbps}$ (the bandwidth leaving node 6 is 3) from $24 \mathrm{Mbps}$. This leaves $21 \mathrm{Mbps}$, which means $21 \mathrm{Mbps}$ of traffic from node 1 sinks into node 6.

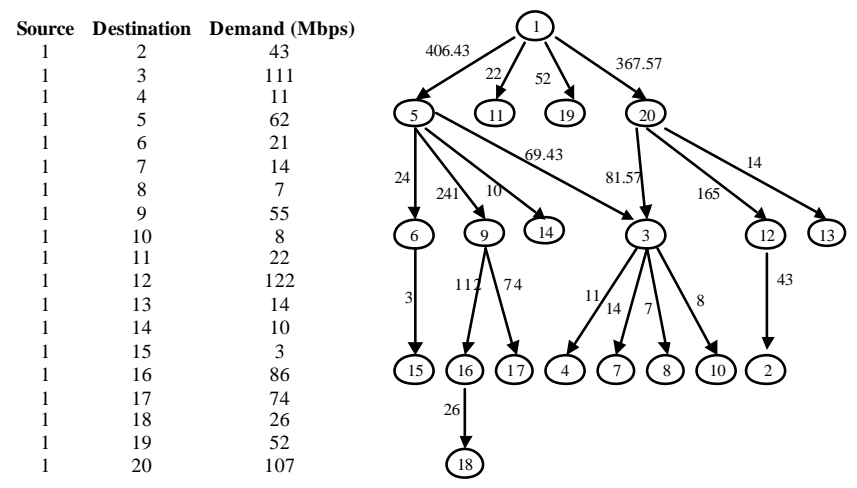

Fig. 2. Part of the forecast $f$ and calculated assigned bandwidth.

After the offline traffic-planning has taken place, the link capacities are broken into two parts: assigned bandwidth $(a b)$ and unassigned bandwidth $(u b)$. Assigned bandwidth represents bandwidth reserved for expected traffic. Unassigned bandwidth refers to free bandwidth for unexpected traffic. From this perspective, the network partially shown in Fig. 2 is called assigned network (assignNet), and the network that consists of unassigned bandwidth is called unassigned network (unassignNet). Note the information of assignNet is local. No information exchange with other routers is required to keep track of the resource consumption within assignNet.

TDWSP also divides incoming QoS requests into two types: expected and unexpected. Expected requests are those that do not exceed the forecasted traffic; otherwise, the requests are deemed unexpected. Accordingly, expected bandwidth $(e b)$ is initialized to the forecast value, e.g., $e b(1,6)=21 \mathrm{Mbps}$.

For a request of bandwidth $b w$ from source node $i$ to destination node $j$, with hop count constraint $c$, the pseudo code for the TDWSP is as follows:

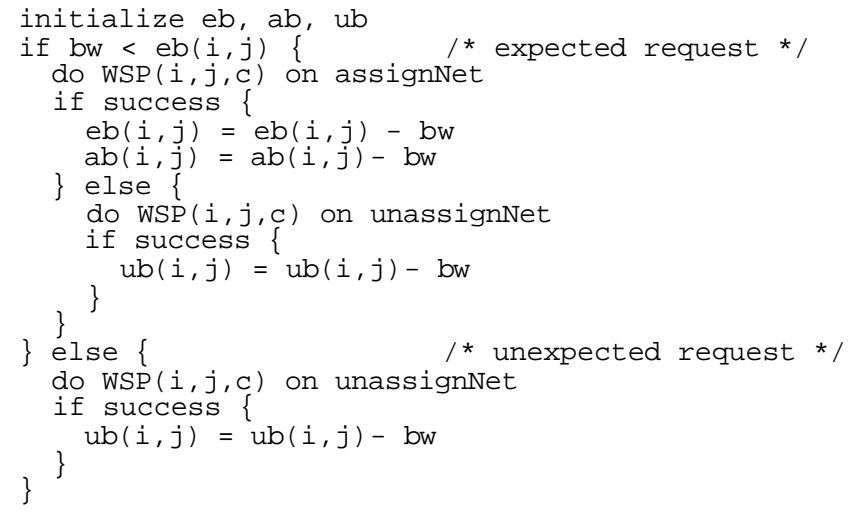

\section{TESTBED}

Two network topologies, network \#1 and \#2, were selected for the simulations. They were real topologies obtained from an ISP in North America. Network \#1 is a 10-node network with 16 bi-directional links that are either OC-12 or OC-48, while network \#2 is a 20-node network with 37 bi-directional links, including OC-3, OC-12 and OC-48. The traffic demands used in the simulations were collected from the ISP as well. Using these two real-world networks gives us realistic insights into the routing issue. In addition, the study was conducted on networks of different sizes to help us better understand the simulation results.

Since routing algorithms are usually sensitive to the sequence of QoS requests, 20 sets of randomly generated requests were tested in every single experiment setting.

An open source implementation of the minimum cost multi-commodity flow algorithm is publicly available as a PPRN package [16]. It is used in this paper to calculate the optimized routing solution. Equation (1) is regarded as the objective function for the offline traffic-planning phase of TDWSP, where $n$ equals 2 . Various values of $n$ were tested in different simulations, and we ascertained that a setting of 2 or 3 is sufficiently good in routing.

\section{THE REAL TRAFFIC PATTERN CASE}

The traffic pattern - the ratio of traffic among the different source-destination pairs - was fixed in the simulations according to the actual measurement from the ISP, while the traffic volume might have been scaled up or down. To make our diagrams more readable, the traffic demand is normalized to the maximum possible value - i.e., $d_{a, 1}$ for network \#1 and $d_{a, 2}$ for network \#2 - that the network can handle. The first subscript of $d$ denotes the specific traffic demand, and the second subscript denotes the network topology number.

The forecast $f$ is set at $d_{b, 1}=53.9 \% d_{a, 1}$ and $d_{b, 2}=50.4 \%$ $d_{a, 2}$ for networks \#1 and \#2, respectively. This means that the forecast is assumed to be at the middle level of network capacity, and can be justified by the fact that current operational networks are normally not running at a high utilization on average [15].

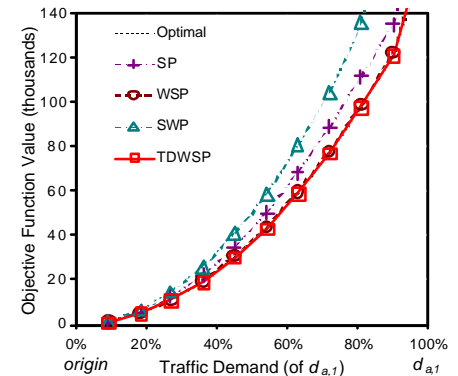

(a) Network $\# 1, f=d_{b, 1}$

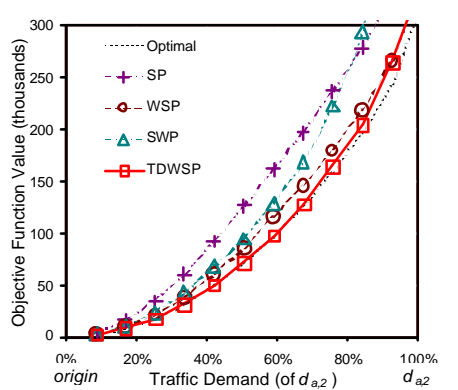

(b) Network \#2, $f=d_{b, 2}$
Fig. 3. Network performance as traffic changes from the origin to $d_{a}$.

In Fig. 3, TDWSP, an example of a time-dependent QoS routing algorithm, outperforms SP, WSP and SWP, examples of state-dependent QoS routing algorithms, at almost all load levels. When the traffic load is below the forecast $f$, the line 
of TDWSP almost sits right on the optimal line. While the actual traffic is higher than $f$, the line of TDWSP leaves the optimal line gradually.

In the 10-node network \#1, TDWSP cannot improve WSP much because it is bounded by the optimal line. However, in the 20-node network \#2, which is a more sophisticated network topology for routing, it is clearer that TDWSP has a strong ability to stay close to the optimal line.

\section{THE ARTIFICIAL TRAFFIC PATTERN CASE}

The simulations presented above were carried out under a real traffic pattern but at different scales. In this section, structurally different traffic demands are considered.

\section{A. The Solvable Traffic Demand Space}

From the routing point of view, this paper defines a solvable traffic demand space as a set of traffic demands with at least one feasible routing solution, no matter whether the feasible routing solution is optimized or not. Because the solvable traffic demand space requires points inside the space to be solvable, the solvable traffic demand space is a determined finite space when the network topology and link capacities are given. Furthermore, it is straightforward to prove that the solvable traffic demand space is convex. In other words, every point on the straight line segment connecting two solvable points $x$ and $y$ is also in the solvable traffic demand space. If the given traffic demand is outside the solvable space, the rejection of part of QoS requests or packet loss is theoretically inevitable.

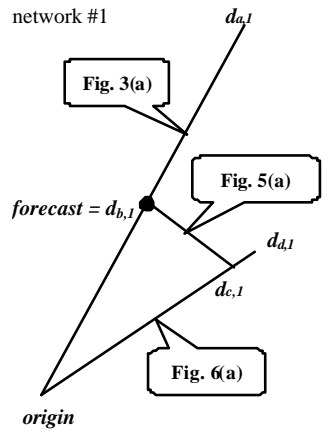

(a) Network \#1, $f=d_{b, 1}$

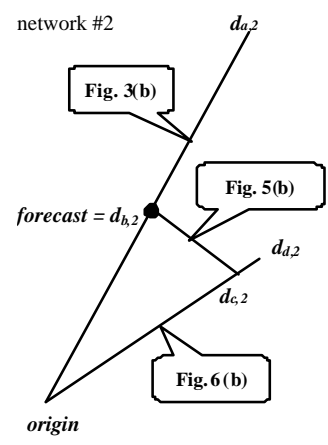

(b) Network \#2, $f=d_{b, 2}$
Fig. 4. Positions of three lines of traffic demand in the solvable space.

In the rest of section VII, we will extend our simulation by testing TDWSP under different traffic demands within the solvable space. Three lines of traffic demand are constructed in the solvable space: The first line runs from the origin to demand $d_{a}$; the second line is from the forecast demand $d_{b}$ to $d_{c}$; and the third line is from the origin to demand $d_{d}$. Their relative positions are illustrated in Fig. 4 . Because $d_{a}, d_{c}$ and $d_{d}$ are all set on the boundary of the solvable space, by changing the traffic demand along these three lines, we intend to investigate the performance of TDWSP in the solvable space more completely.

\section{B. The Line of Traffic Demand from $d_{b}$ to $d_{c}$}

Keeping the total traffic demand level as high as $d_{b, 1}$ and $d_{b, 2}$, two structurally different demands, $d_{c, 1}$ and $d_{c, 2}$, are invented for networks \#1 and \#2, respectively.
Fig. 5 illustrates the performance results for networks \#1 and \#2. The forecast point at $d_{b}$ is configured for TDWSP. The performance of TDWSP is very close to the optimal line. There are two reasons why TDWSP does not degrade much:

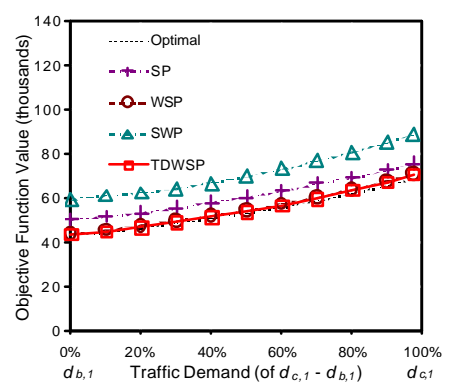

(a) Network $\# 1, f=d_{b, 1}$

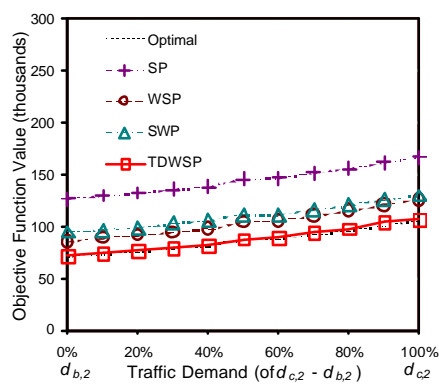

(b) Network \#2, $f=d_{b, 2}$
Fig. 5. Network performance as traffic changes from $d_{b}$ to $d_{c}$.

1) The network-wide traffic load is at about the middle of the network throughput, which is indicated by the mean link utilization of around $35 \%$ and $30 \%$ in networks \#1 and \#2, respectively, based on calculations. When the traffic load is not high, the performance of TDWSP is not sensitive to the configured forecast.

2) The distance from $d_{b}$ to $d_{c}$ is short. For example, in network \#2, the link utilization of all 74 unidirectional links in the optimal solution when the traffic demand is $d_{c, 2}$ is very unevenly distributed. Most links are under-utilized, while a few other links are highly over-utilized. Because of this unbalanced traffic distribution, the distance between $d_{b, 2}$ and $d_{c, 2}$ is short. Any points beyond $d_{c, 2}$ are unsolvable. Because of this short distance, TDWSP does not degrade much before the line goes beyond the solvable space.

\section{The Line of Traffic Demand from $d_{d}$ to the Origin}

A third line from $d_{d}$ to the origin can then be constructed, while the forecast of TDWSP at $d_{b}$ is unchanged.

Because the solvable space is convex, there must be two intersection points or less when one line (from the origin to $d_{c}$ ) penetrates the space. For network \#1, one intersection point is the origin itself, and the other intersection point is at $d_{d, 1}=101 \% d_{c, 1}$. Coincidently, for network \#2, one intersection point is the origin itself, and the other intersection point is at $d_{d, 2}=101 \% d_{c, 2}$.

An important observation here is that in network \#1, the distance from the origin to $d_{a, 1}$ (Euclidean distance $\left|d_{a, 1}\right| \approx$ $3184 \mathrm{Mbps}$ ) is much longer than the dis tance from the origin to $d_{d, 1}\left(\left|d_{d, 1}\right| \approx 1612 \mathrm{Mbps}\right)$, and in network \#2 the distance from the origin to $d_{a, 2}$ is much longer than the distance from the origin to $d_{d, 2}$ (refer to Fig. 4). The key is that both networks \#1 and \#2 are real parts of an ISP's core networks on the Internet, and the demands $d_{a, 1}$ and $d_{a, 2}$ are based on real measurements. It is reasonable to believe that these networks are designed and modified according to real traffic patterns, and therefore they are more suitable for the real traffic pattern than for other artificial traffic patterns, such as $d_{c, 1}$ and $d_{c, 2}$. Usually the mapping of artificial traffic patterns to a real network topology is unbalanced. Therefore, artificial traffic patterns cannot scale well to a large traffic load within 
the solvable traffic demand space.

The simulation results of the third line in networks \#1 and \#2 are satisfactory (Fig. 6). As the demand changes from the origin to $d_{d}$, the curve of TDWSP is extremely close to the optimal curve, although the forecast configuration is based on demands $d_{b, 1}$ and $d_{b, 2}$, respectively.

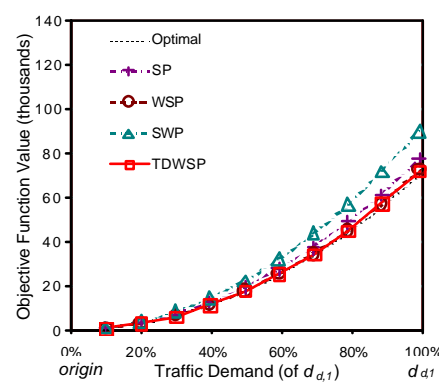

(a) Network \#1, $f=d_{b, 1}$

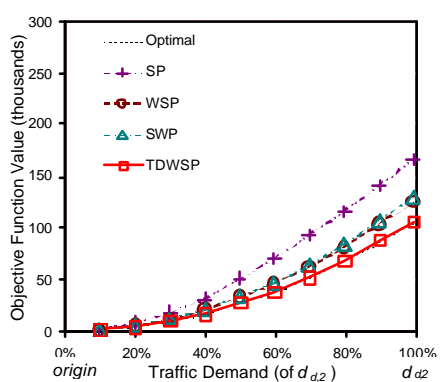

(b) Network \#2, $f=d_{b, 2}$
Fig. 6. Network performance as traffic changes from the origin to $d_{d}$.

\section{CONCLUSIONS}

The benefits of traffic forecasting in the time-dependent mechanism are explained in light of its positive impact on routing performance. In addition, time-dependent mechanism has a few more advantages. Take TDWSP as an example, fewer state updates are required when network resources are allocated and de-allocated, as state updates are triggered only when the real demand exceeds the forecast. Therefore, the stability and scalability problems are relieved. A lower frequency of state updates also decreases the chance that TDWSP will be in an inaccurate state. Thus, TDWSP is more robust than WSP. The routing cost of TDWSP associated with processing state updates and implementing techniques related to the robustness issue is also decreased, so it can respond to a large amount of QoS requests promptly.

Because the performance of TDWSP degrades faster when the actual traffic demand exceeds the forecast, it would be a nice idea to set the forecast at a high level. Nevertheless, if the setting is too high, the time-dependent routing algorithm could lose some of its adaptability to other traffic patterns. The asymmetrical insensitivity does suggest that the extreme value of the daily traffic orbit might be the focus of the traffic forecast. If the routing performance under peak traffic is good, then the routing performance during the rest of the day should be fine.

Our experiments based on real well designed ISP network topologies suggest that a static forecast in TDWSP can accomplish the routing job satisfactorily in a certain range of traffic demands, if traffic demands outside of the solvable space are ignored. Other types of network need to be used for further evaluation in the future.

The results in our paper show that the cost of traffic forecasting in time-dependent QoS routing mechanism could be low. More study on time-dependent routing is advocated.

\section{ACKNOWLEDGMENT}

We would like to thank Ron Armolavicius from Nortel Networks for his feedback on an early outline of this work and Marzia Zaman from Cistel Technology.

\section{REFERENCES}

[1] O. Younis \& S. Fahmy, "Constraint-Based Routing in the Internet: Basic Principles and Recent Research," IEEE Comm. Surveys \& Tutorials, 3rd Quarter 2003.

[2] E. Alitman, T. Basar, T. Jimenez \& N. Shimkin, "Competitive Routing in Networks with Polynomial Cost," Proc. of IEEE INFOCOM 2000, vol. 3, March 2000, pp. 1586-1593.

[3] J. Wang \& K. Nahrstedt, "Hop -by-Hop Routing Algorithms for Premium-Class Traffic in DiffServ Networks," Proc. of IEEE INFOCOM 2002, vol. 2, June 2002, pp. 705-714.

[4] R. Guérin \& A. Orda, "QoS-Based Routing in Networks with Inaccurate Information: Theory and Algorithms," IEEE/ACM Trans. Net., June 1999, pp. 350-364.

[5] G. Apostolopoulos, R. Guérin, S. Kamat, A. Orda \& S. K. Tripathi, "Intra-Domain QoS Routing in IP Networks: A Feasibility and Cost/Benefit Analysis," Special Issue of IEEE Network, vol. 13, no. 5, September 1999.

[6] G. Apostolopoulos, R. Guérin, S. Kamat \& S. Tripathi, "Quality of Service Based Routing: A Performance Perspective," ACM Comp. Commun. Review, 28(4), September 1998.

[7] K. Papagiannaki, N. Taft, Z. Zhang \& C. Diot, "Long-Term Forecasting of Internet Backbone Traffic - Observations and Initial Models," Proc. of IEEE INFOCOM 2003, vol. 2, April 2003, pp. 1178-1188.

[8] A. Elwalid, C. Jin, S. Low \& I. Widjaja, "MATE: MPLS Adaptive Traffic Engineering," Proc. of IEEE INFOCOM 2001, vol. 3, April 2001, pp. 1300-1309.

[9] S. Chen \& K. Nahrstedt, "An Overview of Quality of Service Routing for Next-Generation High-Speed Networks: Problems and Solutions," IEEE Network, vol. 12, no. 6, Nov./Dec. 1998.

[10] A. Juttner, B. Szviatovszki, I. Mecs \& Z. Rajko, "Lagrange Relaxation-Based Method for the QoS Routing Problem," Proc. of IEEE INFOCOM 2001, vol. 2, April 2001, pp. 859868.

[11] T. Korkmaz and M. Krunz, "Multi-Constrained Optimal Path Selection," Proc. of IEEE INFOCOM 2001, vol. 2, April 2001, pp. 834-843.

[12] S. Suri, M. Waldvogel, D. Bauer \& P. R. Warkhede, "Profilebased routing and Traffic Engineering," Computer Communications, vol. 26, 2003, pp. 351-365.

[13] R. Guérin, A. Orda \& D. Williams, "QoS Routing Mechanisms and OSPF Extensions," Proc. of Second Global Internet Miniconference, 1997.

[14] M. Kodialam \& T. V. Lakshman, "Minimum Interference Routing with Applications to MPLS Traffic Engineering," Proc. of IEEE INFOCOM 2000, vol. 2, March 2000, pp. 884893.

[15] A. Odlyzko, "Data Networks are Lightly Utilized, and Will Stay That Way," Technical Report, AT\&T Labs Research, Florham Park, New Jersey, July 1998.

[16] J. Castro \& N. Nabona, "PPRN," Retrieved February 14, 2004, from Statistics and Operations Research Dept. at Universitat Politecnica de Catalunya Web site: http://www-eio.upc.es/ $\sim$ jcastro/pprn.html.

[17] O. Bonaventure, P. Trimintzios, G. Pavlou, et al., "Internet Traffic Engineering," in Quality of Future Internet Services, M. Smirnov et al. (eds), Springer-Verlag, 2003, pp. 118-134.

[18] R. Guérin, H. Ahmadi \& M. Naghshineh, "Equivalent Capacity and Bandwidth Allocation," IEEE Journal on Selected Areas in Communications, September 1991.

[19] M. Schwartz, Broadband Integrated Networks, Prentice-Hall, 1996. 